\title{
Mechanism of protective immunity by vaccination with recombinant Echinococcus granulosus glutathione S-transferase (Chinese strain) in mice
}

\author{
MINGXING ZHU $^{1 *}$, XIUQING WANG ${ }^{2 *}, \mathrm{HAO}^{*} \mathrm{WANG}^{3}, \mathrm{ZHISHENG} \mathrm{WANG}^{4}$, \\ ${\text { JIAQING } \mathrm{ZHAO}^{3} \text {, YANA WANG }}^{1}$ and WEI ZHAO ${ }^{1}$ \\ ${ }^{1}$ Center of Scientific Technology; ${ }^{2}$ Institute of Clinical Laboratory; ${ }^{3}$ School of Basic Medical Sciences; \\ ${ }^{4}$ Experimental Animal Center, Ningxia Medical University, Yinchuan, Ningxia 750004, P.R. China
}

Received August 8, 2014; Accepted May 6, 2015

DOI: $10.3892 /$ etm.2015.2582

\begin{abstract}
The aim of this study was to investigate the immunoprotective effects of recombinant Echinococcus granulosus glutathione S-transferase (rEgGST) against the development of protoscolices (PSCs), and to determine the mechanisms underlying this protection. ICR mice were subcutaneously immunized three times with rEgGST at weeks 0, 2 and 4, followed by the intraperitoneal administration of E. granulosus PSCs at week 10. Six mice in each group were sacrificed at 0 , 2, 4, 6, 10, 18 and 30 weeks following the initial vaccination in order to observe the macroscopic and microscopic effects of parasite development. Various analyses were subsequently conducted, including determination of the levels of immunoglobulins (Igs) and cytokines. Significant differences were observed a number of indices of immune response following immunization with $\mathrm{rEgGST}$. These included reduced cyst formation and elevated levels of IgG1, IgG2a, IgG3, IL-2, IL-4, IL-10 and IFN- $\gamma$, which indicated an increased percentage of immune helper cells. The results of the present study suggest that immunization with rEgGST in mice is able to successfully reduce the $\mathrm{PSC}$-induced formation of cysts and to stimulate an immune response, suggesting that $\mathrm{rEgGST}$ possesses potential value as a candidate vaccine for PSC infection.
\end{abstract}

\section{Introduction}

Human cystic echinococcosis, also known as cystic hydatid disease (CHD), affects humans and livestock and is caused by

Correspondence to: Professor Wei Zhao, Center of Scientific Technology, Ningxia Medical University, Yinchuan, Ningxia 750004, P.R. China

E-mail: zhaoweipaper@126.com

* Contributed equally

Key words: vaccine, immunoprotection, Echinococcus granulosus, recombinant Echinococcus granulosus glutathione S-transferase infection with the larval stage of Echinococcus granulosus. CHD can be seriously harmful to human health and occurs worldwide $(1,2)$. In China, the prevalence of CHD is more extensive than that of alveolar echinococcosis, with 600,000-1,300,000 individuals suffering from CHD (3). At present, the primary methods of treating CHD include early prevention via annual check-ups, medication and surgical operation. However, these approaches are frequently prohibitively expensive, particularly in undeveloped countries and remote areas. Therefore, there is an urgent requirement for highly effective CHD treatments that are relatively inexpensive.

Vaccination of livestock may provide a novel approach for the control of CHD. Previous studies have reported the use of vaccines to effectively protect certain animals, including sheep, goats and bovines, against CHD induced by the cysts of E. granulosus (4-9).

In our previous study, a Chinese strain of E. granulosus glutathione S-transferase (EgGST) was cloned and sequenced (10), and the capacity of EgGST to induce an immune response and immunoprotection was tested in an experimental model of hydatidosis in mice. In the present study, recombinant EgGST (rEgGST) was expressed in Escherichia coli and purified for antigen preparation (11). Following the vaccination of mice with $\mathrm{rEgGST}$, the resulting immunoprotection was analyzed and the protective mechanisms were investigated to assess the potential of $\mathrm{rEgGST}$ as a novel molecular vaccine.

\section{Materials and methods}

Reverse transcription-polymerase chain reaction (RT-PCR). Total RNA was extracted from freshly isolated $E$. granulosus protoscoleces (PSCs) using TRIzol reagent (Invitrogen Life Technologies, Carlsbad, CA, USA). The E. granulosus protoscoleces were extracted aseptically from fertile E. granulosus cysts from the livers and lungs of infected sheep. The EgGST gene was amplified by RT-PCR (Promega Corporation, Madison, WI, USA) using two primers according to the sequence of E. granulosus. Primer I: EcoRI recognition site, 5'-ATGAATTCATGGCTCCCACTCTGGCTT-3'; Primer II: NotI recognition site, 5'-GTGCGGCCGCGTCACCTAACA 
GTCACCAC-3'. Each primer contained EcoRI/NotI restriction enzyme sites and was synthesized by Beijing SBS Genetech, Co., Ltd. (Beijing, China). RT-PCR was performed in a 50- $\mu 1$ reaction mixture, containing $10 \mu \mathrm{l} 5 \mathrm{X}$ buffer (Promega Corporation), $2 \mu 1 \mathrm{Mg}^{2+}(25 \mathrm{mmol} / \mathrm{l}), 1 \mu \mathrm{ldNTP}(10 \mathrm{mmol} / \mathrm{l})$, $1 \mu 1$ avian myeloblastosis virus reverse transcriptase (Promega Corporation), $1 \mu \mathrm{l}$ Thermus flavus DNA polymerase (Promega Corporation), $5 \mu 1$ of each primer, $5 \mu 1$ RNA and $20 \mu 1$ diethylpyrocarbonate-treated water. The reaction protocol for RT-PCR was as follows: $48^{\circ} \mathrm{C}$ for $45 \mathrm{~min}, 94^{\circ} \mathrm{C}$ for $2 \mathrm{~min}$, followed by 40 cycles of $30 \mathrm{sec}$ at $94^{\circ} \mathrm{C}, 60 \mathrm{sec}$ at $60^{\circ} \mathrm{C}$ and $2 \mathrm{~min}$ at $68^{\circ} \mathrm{C}$, with a final extension for $7 \mathrm{~min}$ at $68^{\circ} \mathrm{C}$. RT-PCR products were identified using $1 \%$ agarose gel electrophoresis (Liuyi Instrument Factory, Beijing, China).

Subcloning of EgGST gene into expression plasmid vector. The target fragment was purified using a gel cleanup kit (SBS Genetech, Co., Ltd.) and inserted between the EcoRI and NotI sites of the expression vector pET-28a (Novagen; Merck KGaA, Darmstadt, Germany). The recombinant vector EgGST/pET-28a was identified by restriction digestion using EcoRI and NotI and the EgGST insert was verified by sequencing, performed by Sangon Biotech Co., Ltd. (Shanghai, China). E. coli BL21 (DE3) pLysS, provided by Dr Xiao Wei (University of Saskatchewan, Saskatoon, Canada) was transformed for induced expression of His6-tagged EgGST protein.

Expression and purification of $r$ Eg GST. Protein expression was induced at $25^{\circ} \mathrm{C}$ by cultivation of the transformed E. coli BL21 overnight in the presence of isopropyl- $\beta$-D-thiogalactoside (IPTG; Promega Corporation) at a final concentration of $0.6 \mathrm{mmol} / \mathrm{l}$. The recombinant His6-tagged $\mathrm{rEgGST}$ was purified from the extract of transformed E. coli $\mathrm{BL} 21$ (DE3) by $\mathrm{Ni}^{2+}$ chelate affinity chromatography (Novagen) according to the manufacturer's instructions. Purified His6-tagged protein was analyzed using $12 \%$ sodium dodecyl sulfate-polyacrylamide gel electrophoresis (SDS-PAGE) gel. Protein concentrations were determined using the Bradford method (12).

Immunization. A total of 84 male 6-week old ICR mice were obtained from the Experimental Animal Centre of Ningxia Medical University (Yinchuan, China). Mice were allocated at random into two groups containing 42 mice each. Mice in group A received three subcutaneous immunizations with $10 \mu \mathrm{g}$ rEgGST in $100 \mu \mathrm{l}$ phosphate-buffered saline (PBS) emulsified in Freund's adjuvant (Sigma-Aldrich, St. Louis, MO, USA). The three immunizations were delivered at 2-week intervals, starting at week 0 in Freund's complete adjuvant and followed by two booster immunizations in Freund's incomplete adjuvant at weeks 2 and 4 . Mice in the control group B were injected with the corresponding adjuvant and PBS. This study was approved by the Ningxia Medical University Ethical Committee.

Challenge infection and protective immunity. Six weeks after the final vaccination, on week 10 , a challenge infection was induced in the mice via the intraperitoneal injection of 1,500 PSCs. Six mice in each group were sacrificed at different $0,2,4,6,10,18$ and 30 weeks following the initial vaccination, in order to obtain sera and spleen cells. Mice were sacrificed by cervical vertebra dislocation. Subsequently, the carcasses were dissected and examined superficially for visible hydatid cysts. The percentage of protection was determined according to the method and formula described by Dempster et al (13): Protective immunity in vaccinated mice $(\%)=$ (average number of cysts in the test group/average number of cysts in the control group) x 100.

Serum collection. Six mice in each group were sacrificed at 0 , $2,4,6,10,18$ and 30 weeks following the initial vaccination in order to observe the macroscopic and microscopic effects of parasite development. Serum samples were collected and stored individually at $-84^{\circ} \mathrm{C}$.

Cytokine measurements using ELISA. The optical density values of a number of cytokines were determined using ELISA. Spleens were isolated from mice and splenocytes were harvested. A suspension of single splenocytes was prepared after removing erythrocytes via hypotonic lysis and resuspending the samples in RPMI 1640 (Gibco Life Technologies, Carlsbad, CA, USA) by vigorous pipetting. Viable cells counted by trypan blue exclusion $\left(5 \times 10^{6}\right.$ cells $\left./ \mathrm{ml}\right)$ were exposed to medium, $5 \mu \mathrm{g} / \mathrm{ml}$ concanavalin A (Sigma-Aldrich) and $10 \mu \mathrm{g} / \mathrm{ml} \mathrm{rEgGST}$ and incubated for $72 \mathrm{~h}$. Supernatants of lymphocyte cultures were added to a pre-coated microplate ELISA kit (Jingmei Biotech Co., Ltd., Shenzhen, China) and maintained at $37^{\circ} \mathrm{C}$ for $2 \mathrm{~h}$. After washing with PBS and 0.05\% Tween 20 (PBST), 25-50 ng goat biotin-conjugated antibodies against mouse IL-2 (F10092-A), IL-4 (F7695-A), IL-10 (F7701-A) and IFN- $\gamma$ (F10077-A; 1:1000; Jingmei Biotech Co. Ltd.) were added per well and the plates were incubated for $2 \mathrm{~h}$ at $37^{\circ} \mathrm{C}$. Following four washes in PBST, peroxidase-labeled streptavidin was added for $1.5 \mathrm{~h}$ at $37^{\circ} \mathrm{C}$. Plates were washed and incubated with substrate for $0.5 \mathrm{~h}$ at $37^{\circ} \mathrm{C}$. Finally, the reaction was stopped with $100 \mathrm{ml}$ sulfuric acid (2 M). Optical density was measured at $450 \mathrm{~nm}$ using an ELISA reader (Bio-Rad Laboratories, Inc., Hercules, CA, USA).

Antibody measurement using ELISA. Serum antibody levels were quantified using ELISA at 0, 2, 4, 6, 10, 18 and 30 weeks after the first of the three immunizations (14). In this assay, 96-well microtiter plates (Sino-American Biotechnology Co., San Diego, CA, USA) were coated with $\mathrm{rEgGST}(10 \mu \mathrm{g} / 100 \mu \mathrm{l} / \mathrm{well})$ and incubated overnight in $0.1 \mathrm{M}$ carbonate buffer ( $\mathrm{pH} 9.6)$ at $4^{\circ} \mathrm{C}$. Serum samples were diluted 1:100 in PBST and tested in duplicate. Bound antibodies were detected using HRP-conjugated goat anti-mouse IgG (ab97265) and IgG subclass antibodies against IgG1 (ab97240), IgG2a (ab97245) and IgG3 (ab97260; Abcam, Cambridge, UK) at a 1:1,000 dilution in PBST. Antibody titers were measured at $450 \mathrm{~nm}$ using an ELISA reader (Bio-Rad Laboratories, Inc.).

Statistical analysis. All data comparisons were tested for significance using one-way analysis of variance. SPSS software, version 17.0 (SPSS, Inc., Chicago, IL, USA) was used to perform statistical analyses. $\mathrm{P}<0.05$ was considered to indicate a statistically significant difference. 
Table I. Number of hydatid cysts and protective immunity in vaccinated and control groups.

\begin{tabular}{lccc}
\hline Group & Mice (n) & Cysts (n) & Immunity (\%) \\
\hline Control & 6 & $3.67 \pm 3.14$ & - \\
rEgGST & 6 & $0.33 \pm 0.51^{\mathrm{a}}$ & $89.39^{\mathrm{a}}$ \\
\hline
\end{tabular}

Data are expressed as mean \pm standard deviation. ${ }^{\text {a }}<0.01$ vs. the control group. rEgGST, recombinant Echinococcus granulosus glutathione S-transferase.

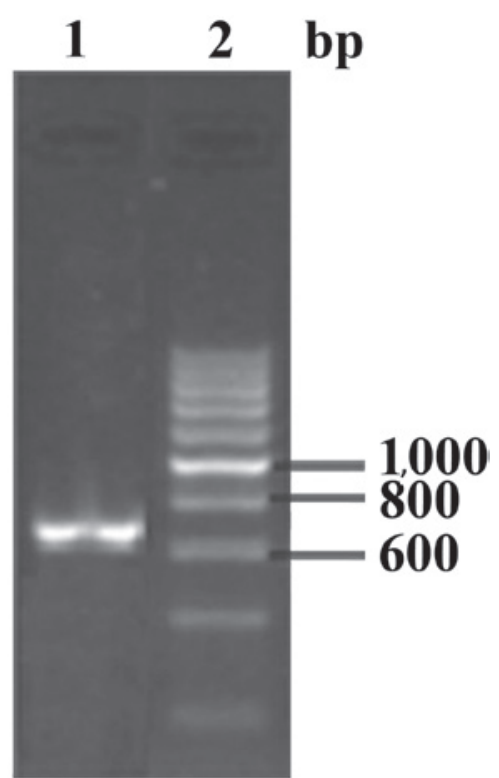

Figure 1. Amplified product of the glutathione S-transferase gene. Lane 1, polymerase chain reaction product; lane 2, DNA marker.

\section{Results}

Expression and purification of $r E g G S T$. The EgGST gene was amplified using RT-PCR, generating an amplified product of $\sim 660$ bp (Fig. 1). The recombinant expression plasmid EgGST/pET-28a was constructed and transformed into E. coli BL21 (DE3). The plasmids extracted from transformed E. coli BL21 (DE3) were digested using the restriction enzymes EcoRI and NotI (Fig. 2). The insert sequence was analyzed using DNA sequencing and confirmed to be the EgGST gene, suggesting that the recombinant plasmid EgGST/pET-28a had been constructed successfully. Following IPTG induction, the His6-labeled recombinant protein was purified using a $\mathrm{Ni}^{2+}$-chelating column. SDS-PAGE staining results demonstrated that the His6-tagged EgGST protein had been successfully expressed in E. coli BL21 (DE3) and purified efficiently from the $E$. coli lysate, with a molecular weight of $\sim 28 \mathrm{kD}$ (Fig. 3).

Antibody measurement using ELISA. Sera from animals treated with PBS or rEgGST were tested using the ELISA method. The levels of IgG were increased following rEgGST immunization, typically from about week 4 after the initial immunization until week 10 . The animals were challenge-infected at

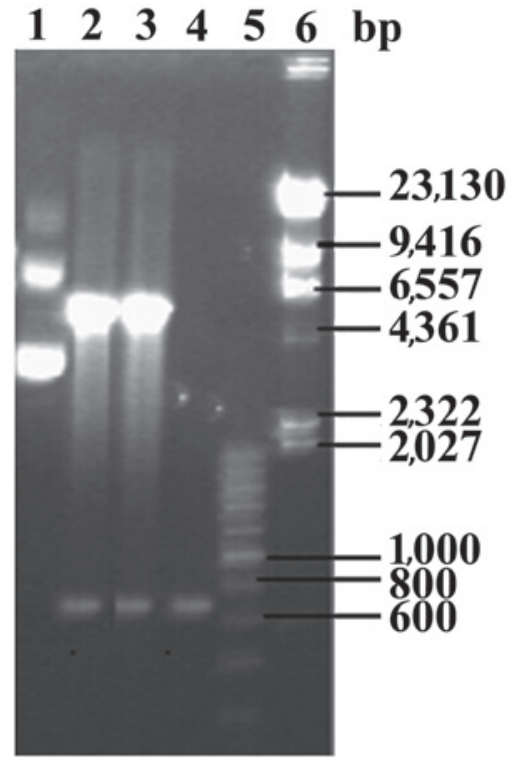

Figure 2. Restriction enzyme digestion of recombinant expressed plasmid Echinococcus granulosus glutathione S-transferase (GST)/pET-28a by EcoRI and NotI. Lane 1, recombinant plasmid; lane 2-3, GST/pET-28a digested by EcoRI and NotI; lane 4, polymerase chain reaction-amplified product; lane 5, 200-bp DNA marker; lane 6, $\lambda$ DNA/HindIII marker.

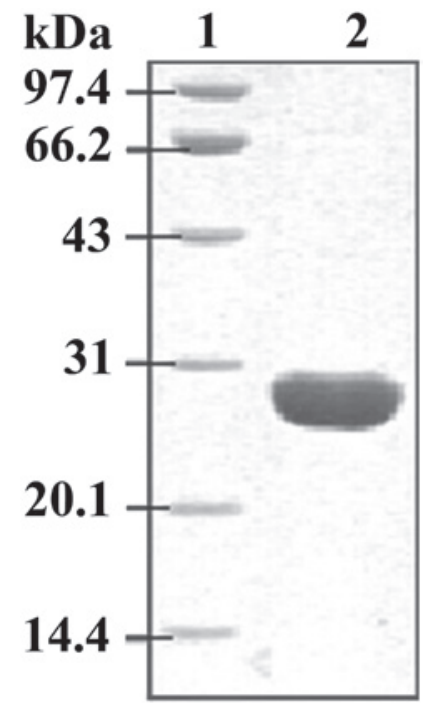

Figure 3. SDS-PAGE analysis purification of recombinant Echinococcus granulosus glutathione S-transferase (rEgGST). Lane 1, molecular weight standards marker; lane 2, purified rEgGST protein.

week 10. Following the challenge infection, the levels of total IgG remained elevated (Fig 4A), the levels of IgG2a and IgG3 declined gradually (Fig. 4C and D), and the levels of IgG1 continued to increase until week 30 (Fig. 4B).

Analysis of cytokine levels in mice immunized with $r E g G S T$ by ELISA. The animals' reaction to $\mathrm{rEgGST}$ was further evaluated by measuring the levels of a number of cytokines, specifically interferon (IFN)- $\gamma$, interleukin (IL)-2, IL-4 and IL-10. The levels of IFN- $\gamma$ and IL-2 in the immunized group were significantly higher compared with those in the control group at week 6 after the first immunization, while the levels of IL-4 and IL-10 remained unaltered during the period of observa- 
A

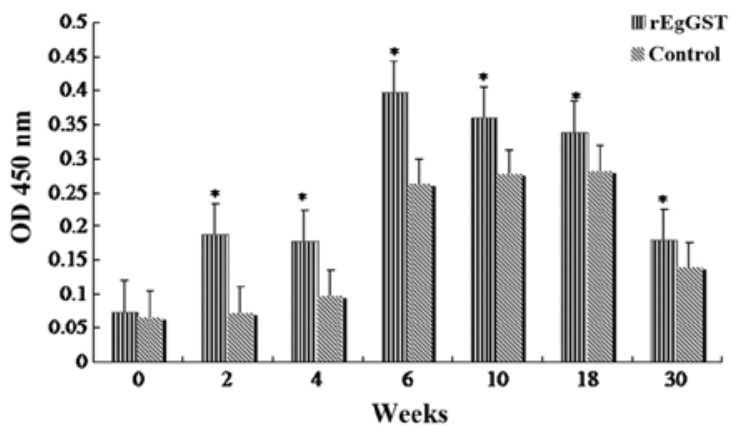

C

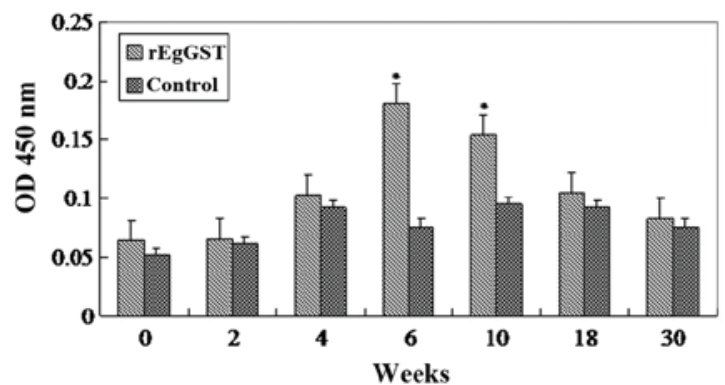

B
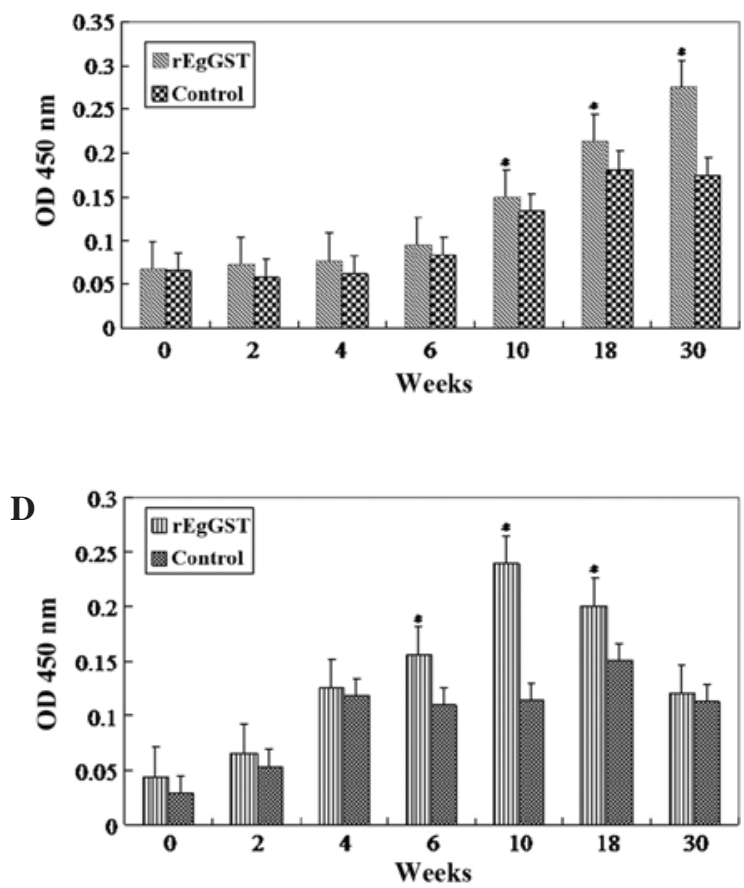

Figure 4. Total IgG antibody and IgG subclass response as detected by ELISA analysis of sera. (A) IgG, (B) IgG1, (C) IgG2a and (D) IgG3 response. Different stages were selected for antibody examination: Week 0 (before the first immunization), week 2 (second immunization), week 4 (third immunization), week 6 , week 10 (challenge infection), week 18 and week 30. Data are presented as the mean \pm standard error of the mean of 6 mice per group. * $<<0.05$ vs. control. rEgGST, recombinant Echinococcus granulosus glutathione S-transferase; OD, optical density.

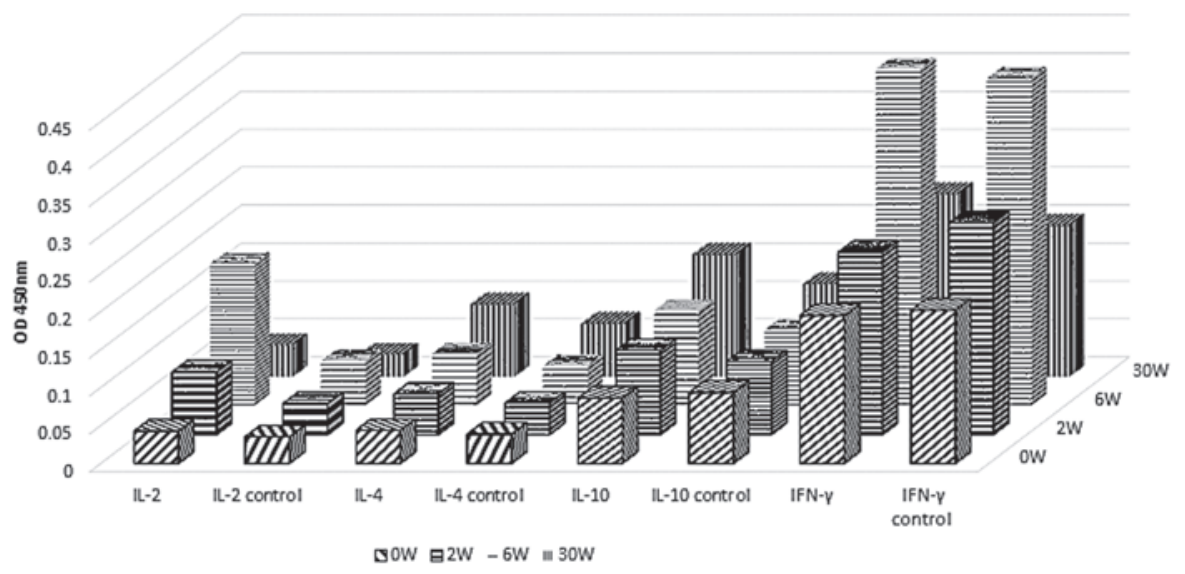

Figure 5. Detection of cytokines in mice at various time points following vaccination. OD, optical density; IL, interleukin; IFN, interferon.

tion. After the challenge infection at week 10, the levels of IFN- $\gamma$ and IL-2 decreased to normal levels by week 30 . The levels of IL-4 and IL-10 did not change significantly following the challenge infection in the immunization group (Fig. 5). These results indicate that type $1 \mathrm{~T}$ helper (Th1) cells mediate the primary response following immunization.

Protective immunity in mice. Mice in each group were sacrificed at 5 months (30 weeks) after the initial immunization. The internal organs of the mice were examined for the presence of hydatid cysts. Mice vaccinated with $\mathrm{rEgGST}$ exhibited significantly reduced numbers of hydatid cysts compared with the control group (Table I; P<0.01). The protective immunity induced by $\mathrm{rEgGST}$ was calculated to be $89.39 \%$.

\section{Discussion}

Previous studies have reported that the 14-3-3 protein (15), myophilin (16), ferritin (17) and P29 (18) that are secreted by E. granulosus exhibit marked immunogenic properties. The aim of the present study was to determine whether immunization with $\mathrm{rEgGST}$ was able to induce effective protective immunity when compared with a control group. A positive result may be used to provide an experimental basis for the potential use of rEgGST as a vaccine. 
Glutathione S-transferases (GSTs) are a family of detoxification enzymes (19), and Schistosoma mansoni $28 \mathrm{kDa}$ GST (Sj28GST) has been recognized as an effective protective antigen against S. mansoni (20). For E. granulosus, the observation of GST protein expression at various stages of parasite development provided an experimental basis for the investigation of the potential of EgGST as a vaccine candidate against echinococcosis (21). Therefore, GST is considered to be a potential treatment for the prevention of shistosomiasis infection (22).

In the present study, immunization with rEgGST stimulated humoral immunity in mice following the third immunization. Increased serum levels of total $\mathrm{IgG}$ and the IgG subtypes $\operatorname{IgG} 2 \mathrm{a}$, IgG1 and $\mathrm{IgG} 3$ were observed in mice following rEgGST immunization and challenge infection compared with the control group. These results indicate that an immune response may be effectively activated by treatment with $\mathrm{rEgGST}$. In addition, IgG, IgG2a, IgG1 and IgG3 may be involved in the early protective immune response. As the time following the induction of the challenge infection increased, IgG2a and IgG3 levels decreased gradually while the levels of IgG1 increased continuously. This observation may be related to the parasite escaping from host immune surveillance. rEgGST was able to induce the production of high levels of antibody following immunization, which remained elevated following early infection. These results indicate that rEgGST possesses notable immunogenicity and immunoreactivity.

Cytokines are commonly produced by $\mathrm{T}$ helper cells in humoral and cellular immunity in order to mediate the regulation of parasitic diseases. Th1 cells produce interleukin (IL) -2 and interferon (IFN) $-\gamma$ to promote $\operatorname{IgG} 2 \mathrm{a}$ and $\mathrm{IgG} 3$ production. Th2 cells produce IL-4 and IL-10 and promote a humoral response, in particular via the induction of IgG1. The Th1 protective response mediates protective immunity and helps the host to eliminate hydatid disease, while the Th2 response promotes the humoral immune response and mitigates parasitism (23-25). In the present study, four cytokines were selected (IFN- $\gamma$, IL-2, IL-10 and IL-4) for examination of their dynamic changes in mice following treatment with rEgGST and the induction of a challenge infection using PSCs. Following rEgGST immunization, the levels of Th1-type cytokines (IFN- $\gamma$ and IL-2) significantly increased; however, the levels of Th2-type cytokines (IL-4 and IL-10) remained unaltered. Levels of Th1-type cytokines decreased following the induction of the PSC challenge infection, and the levels of Th2-type cytokines remained the same. These results suggest that rEgGST induces the Th1-type immune response, which gradually transitions into the Th2-type response at later stages of infection. The changes in cytokines levels are typically associated with the changes in the levels of specific antibodies. It was observed that following rEgGST immunization, the Th1-dependent increase of IgG2a and IgG3 levels was coordinated with the increase of Th1-type cytokines. However, the increase of Th2-dependent IgG1 was not associated with an increase of Th2 type cytokines. These results suggest that rEgGST immunization specifically stimulates Th1 cells, which subsequently induce the production of Th1-dependent antibodies and Th1-type cytokines. However, the mechanism by which $\mathrm{rEgGST}$ stimulates the observed immune response in animals remains unclear.
In conclusion, the results of the present study demonstrate that $89.39 \%$ protection may be induced by administering rEgGST in a murine model of echinococcosis. Therefore, rEgGST is potentially competent to be used as the anti-hydatid component of a novel vaccine candidate, with the aim of effectively preventing and controlling of the development of hydatid disease. However, the mechanism underlying the observed rEgGST-induced protection remains unclear and requires further study.

\section{Acknowledgements}

This study was supported by a grant from the National Natural Science Foundation of China (no. 81360249).

\section{References}

1. Zhang W and McManus DP: Vaccination of dogs against Echinococcus granulosus: A means to control hydatid disease? Trends Parasitol 24: 419-424, 2008.

2. Battelli G: Echinococcosis: Costs, losses and social consequences of a neglected zoonosis. Vet Res Commun 33 (Suppl 1): 47-52, 2009.

3. Craig PS; Echinococcosis Working Group in China: Epidemiology of human alveolar echinococcosis in China. Parasitol Int 55 (Suppl): S221-S225, 2006.

4. Heath DD and Lawrence SB: Antigenic polypeptides of Echinococcus granulosus oncospheres and definition of protective molecules. Parasite Immunol 18: 347-357, 1996.

5. Lightowlers MW, Lawrence SB, Gauci CG, Young J, Ralston MJ, Maas D and Health DD: Vaccination against hydatidosis using a defined recombinant antigen. Parasite Immunol 18: 457-462, 1996.

6. Dempster RP, Robinson CM and Harrison GB: Parasite vaccine development: Large-scale recovery of immunogenic Taenia ovis fusion protein GST-45W(B/X) from Escherichia coli inclusion bodies. Parasitol Res 82: 291-296, 1996.

7. Manderson D, Dempster R and Chisti Y: A recombinant vaccine against hydatidosis: Production of the antigen in Escherichia coli. J Ind Microbiol Biotechnol 33: 173-182, 2006.

8. Larrieu E, Herrero E, Mujica G, et al: Pilot field trial of the EG95 vaccine against ovine cystic echinococcosis in Rio Negro, Argentina: Early impact and preliminary data. Acta Trop 127: 143-151, 2013.

9. Heath DD, Robinson C and Lightowlers MW: Maternal antibody parameters of cattle and calves receiving EG95 vaccine to protect against Echinococcus granulosus. Vaccine 30: 7321-7326, 2012.

10. Li Z, Zhao J, Wang Y, et al: Cloning and sequence analyzing of glutathione S-transferase gene on Echinococcus granulosus of China. Ning Xia Yi Xue Yuan 28: 4-6, 2006 (In Chinese).

11. Gao Peng, Xiong Ying, Du Juan, et al: Immune protection of recombinant protein glutathione $\mathrm{S}$-transferase (rEgGST) of Echinococcus granulosus (Chinese mainland strain). Zhong Guo Wei Sheng Wu Xue Hui 27: 238-240, 2011 (In Chinese).

12. Bradford MM: A rapid and sensitive method for the quantitation of microgram quantities of protein utilizing the principle of protein-dye binding. Anal Biochem 72: 248-254, 1976.

13. Dempster RP, Berridge MV, Harrison GB and Heath DD: Echinococcus granulosus: Development of an intermediate host mouse model for use in vaccination studies. Int J Parasitol 21: 549-554, 1991.

14. Liu JM, Wang AH, Chen YJ and Gu HM: Studies on the antigenicity and protein pattern of soluble antigens from a germinal cell line of Echinococcus granulosus. Nong Ye Sheng Wu Ji Shu Xue Bao 6: 277-280, 1998 (In Chinese).

15. Li ZJ, Wang YN, Wang Q and Zhao W: Echinococcus granulosus 14-3-3 protein: A potential vaccine candidate against challenge with Echinococcus granulosus in mice. Biomed Environ Sci 25: 352-358, 2012.

16. Sun J, Wang Y, Li Z, et al: Echinococcus granulosus: Immunoprotection accompanied by humoral and cytokine response against secondary hydatidosis in mice immunized with rEg.myophilin. Vet Res Commun 35: 193-200, 2011. 
17. Wang Y, Li ZJ, Li ZY, Bo Y and Zhao W: Recombinant ferritin protects mice against challenge with Echinococcus granulosus? Acta Parasitological 54: 335-340, 2009.

18. Shi Z, Wang Y, Li Z, et al: Cloning, expression, and protective immunity in mice of a gene encoding the diagnostic antigen P-29 of Echinococcus granulosus. Acta Biochim Biophys Sin (Shanghai) 41: 79-85, 2009.

19. Bhargavi R, Vishwakarma S and Murty US: Modeling analysis of GST (glutathione-S-transferases) from Wuchereria bancrofti and Brugia malayi. Bioinformation 1: 25-27, 2005.

20. Rezende CM, Goes TS, Goes VS, Azevedo V, Leite MF and Goes AM. GM-CSF and TNF-alpha synergize to increase in vitro granuloma size of PBMC from humans induced by Schistosoma mansoni recombinant $28-\mathrm{kDa}$ GST. Immunol Lett 95: 221-228, 2004.

21. Zheng H, Zhang W, Zhang L, et al: The genome of the hydatid tapeworm Echinococcus granulosus. Nat Genet 45: 1168-1175, 2013.
22. Wilson MS, Mentink-Kane MM, Pesce JT, et al: Immunopathology of schistosomiasis. Immunol Cell Biol 85: 148-154, 2007.

23. Shainheit MG, Saraceno R, Bazzone LE, et al: Disruption of interleukin-27 signaling results in impaired gamma interferon production but does not significantly affect immunopathology in murine schistosome infection. Infect Immun 75: 3169-3177, 2007.

24. Nono JK, Lutz MB and Brehm K: EmTIP, a T-Cell immunomodulatory protein secreted by the tapeworm Echinococcus multilocularis is important for early metacestode development. PLoS Neg1 Trop Dis 8: e2632, 2014.

25. Boutennoune H, Qaqish A, Al-Aghbar M, et al: Induction of $\mathrm{T}$ helper 1 response by immunization of $\mathrm{BALB} / \mathrm{c}$ mice with the gene encoding the second subunit of Echinococcus granulosus antigen B (EgAgB8/2). Parasite 19: 183-188, 2012. 